\title{
The Role of Crossing Symmetry for a Low Energy Pion-Nucleon Scattering from Dispersive Method
}

\author{
Walter Luiz Alda Jr* and J. Sá Borges ${ }^{\dagger}$ \\ Universidade do Estado do Rio de Janeiro \\ 20550-013, Rio de Janeiro, RJ, Brazil
}

(Received on 1 April, 2008)

\begin{abstract}
The slow convergence of chiral perturbation theory for heavy baryons (HBChPT) suggests that any attempt to unitarize the amplitude following from this method will fail to describe the experimental phase shifts. However, it was possible to obtain a ChPT pion nucleon amplitudes respecting exact unitarity relation by using the Inverse Amplitude Method (IAM), but the resulting total amplitude violates the important property of crossing symmetry [1] . On the other hand, the use of a dispersive calculation, starting directly from a result at second order in the pion momentum, is an alternative approach to get unitarized scattering amplitude. By this method it was possible to fit, with two parameters, the $P_{33}$ partial wave to the experimental low energy phase shifts, and to present the resulting $S$ and $P$ partial wave phase shifts [2]. This was done with a crossing symmetric amplitude, that respect approximate elastic unitarity relation. In the present exercise, we do not impose crossing symmetry for the amplitude obtained in the previous work, in order to verify the role played by crossing symmetry in the dispersive approach. As in the previous work, our strategy was to perform a fit of the $P_{33}$ amplitude to the experimental phase shifts and then use the fixed parameters in the $S$ and $P$ partial waves to compare them with the corresponding experimental phase shifts. We conclude that, when we do not impose crossing symmetry for the total amplitude, more parameters are needed in the fitting procedure for $P_{33}$, moreover the theoretical results for $S_{11}, S_{31}, P_{11}, P_{31}$ and $P_{13}$ are quite far from the experimental points.
\end{abstract}

Keywords: Chiral Symmetry; Dispersion Relations; Partial-wave analysis

\section{INTRODUCTION}

Low energy hadron physics must still be described by phenomenology even though nowadays the Quantum Chromodynamics (QCD) is considered the correct description of the strong interactions. A great theoretical improvement was made by means of the method of Chiral Perturbation Theory (ChPT) [3], which is an effective theory derived from the basis of QCD. The method consists of writing down chiral Lagrangian for the physical processes and uses the conventional technique of the field theory for the calculations.

In order to deal with baryons the theory faced problems related to fixing the scale for momenta and quark mass expansion and one issue known as Heavy Barion Chiral Perturbation Theory have been applied for instance to describe pionnucleon scattering [4]. The method yields a total amplitude and the corresponding partial waves satisfy approximate elastic unitarity relation because the leading amplitude is a real function of energy on the physical region. This lack of unitarity calls for an unitarization method.

In another context, namely the hard-meson method of current algebra, it was possible to obtain a low energy pion nucleon real amplitude in second order in pion momentum, reproducing Weinberg prediction for S-wave scattering length [5]. Clearly this result also calls for some unitarization procedure. The unitarization program of current algebra (UPCA), where one starts from an approximate low energy amplitude in order to construct corrected amplitudes, was applied to this process[2]. The crossing symmetry property for the total

\footnotetext{
*Electronic address: walteralda@gmail.com
}

${ }^{\dagger}$ Electronic address: saborges@uerj.br
Dirac amplitudes was imposed and auxiliary functions have been constructed in order to respect approximate unitarity relation. The method consider known the imaginary part of partial-wave amplitudes as given by the lowest order amplitude and uses the dispersion relation technique in order to arrive at a quasi-unitarized amplitude written in terms of known functions and a two free parameters polynomial part in energy.

The strategy was to fit of the isospin $3 / 2 P$-wave phaseshifts using two available parameters. Having fixed these two parameters, one looks at the resulting phase-shifts for $S_{11}$, $S_{31}, P_{11}, P_{13}$ and $P_{31}$. Some model phase-shifts were seen to keep similarities with the experimental results. The fact that the violation of unitarity of the resulting amplitudes are large, mainly in the resonance region, claims for a next order approximation by the dispersion relation technique, using the tools given in [2].

Instead of going to the next order of the calculation, our aim in the present work is to verify the importance of crossing symmetry for the amplitudes presented in the previous work. This way, in the present work, the Dirac amplitudes depend on $s$ and $t$ Mandelstan variables and we do not add any $u$ dependence for the total amplitudes.

In the next section we present the basic formalism and the amplitude that we started from to construct first order corrected in unitarity amplitudes. We show the fit of $P_{33}$ to experimental phase shifts, by fixing three available parameters. Once fixed the parameters, we compare the resulting $S_{11}, S_{31}$, $P_{11}, P_{13}$ and $P_{31}$ phases with those corresponding to the amplitudes that respect the crossing symmetry constraint. Finally we present some conclusions. 


\section{BASIC FORMALISM}

We will consider the T-matrix for the reaction $\pi(k)+$ $N(p) \rightarrow \pi\left(k^{\prime}\right)+N\left(p^{\prime}\right)$

$$
T^{ \pm}\left(p^{\prime}, k^{\prime} ; p, k\right)=-A^{ \pm}(s, t, u)+\frac{i}{2}\left(k+k^{\prime}\right) B^{ \pm}(s, t, u),
$$

The Dirac amplitudes exhibit the following symmetry properties under crossing:

$$
A^{ \pm}(s, t, u)= \pm A^{ \pm}(u, t, s) \quad \text { and } \quad B^{ \pm}=\mp B^{ \pm}(u, t, s) ;
$$

the amplitudes corresponding to definite isospin $1 / 2$ and $3 / 2$ are given by:

$$
A_{1 / 2}=A^{+}+2 A^{-}, A_{3 / 2}=A^{+}-A^{-}
$$

and similarly for $B$.

We work in the center of mass system, so that the four momenta are defined as $k=(\vec{k}, w), k^{\prime}=\left(\vec{k}^{\prime}, w\right), \quad p=$ $(-\vec{k}, E)$ and $p^{\prime}=\left(-\overrightarrow{k^{\prime}}, E\right)$, with $|\vec{k}|=\left|\overrightarrow{k^{\prime}}\right|, w=\sqrt{\vec{k}^{2}+m^{2}}$ and $E=\sqrt{\vec{k}^{2}+M^{2}}, M$ and $m$ being the nucleon and the pion mass, respectively. The total energy and scattering angle are given by $W=E+w$ and $|\vec{k}|^{2} \cos \theta=\vec{k} \cdot \vec{k}^{\prime}$, thus, in terms of these quantities, one has $s=W^{2}, t=-2|\vec{k}|^{2}(1-\cos \theta)$.

For each isospin $I$ the Pauli amplitudes are:

$$
\begin{gathered}
F_{1 I}=\frac{E+M}{8 \pi W}\left[A_{I}(s, \cos \theta)+(W-M) B_{I}(s, \cos \theta)\right], \\
F_{2 I}=\frac{E-M}{8 \pi W}\left[-A_{I}(s, \cos \theta)+(W+M) B_{I}(s, \cos \theta)\right] .
\end{gathered}
$$

Partial wave amplitudes $f_{I \ell}^{ \pm}$are defined as

$$
\begin{gathered}
f_{I \ell}^{ \pm}(s)=F_{1 I \ell}(s)+F_{2 I \ell \pm 1}(s), \quad \text { where } \\
F_{i I \ell}(s)=\frac{1}{2} \int_{-1}^{+1} F_{i I}(s, x) P_{\ell}(x) d x \\
\text { for } i=1,2 \text { and } I=1 / 2,3 / 2 .
\end{gathered}
$$

For elastic scattering we have

$$
\operatorname{Im} f_{I \ell}^{ \pm}(s)=|\vec{k}|\left|f_{I \ell}^{ \pm}(s)\right|^{2}
$$

which may be solved yielding

$$
f_{I \ell}^{ \pm}(s)=1 /|\vec{k}| e^{i \delta_{I \ell}(s)} \sin \delta_{I \ell}(s),
$$

where $\delta_{I \ell}(s)$ are real phase shifts.

The quasi-unitarized amplitude satisfies approximate unitarity and, in this case, the definition of phase-shifts is quite arbitrary. We will adopt the following definition

$$
\delta_{I \ell}=\tan ^{(-1)} \frac{\operatorname{Im} f_{I \ell}}{\operatorname{Re} f_{I} \ell}
$$

\section{UNITARIZATION}

Up to first-order in pion momenta, the amplitudes leading to the well known Weinberg prediction for $S$ - wave scattering lengths are:

$$
A^{(0)-}=\frac{\mu_{V}}{8 M f^{2}}(u-s) \quad \text { and } \quad B^{(0)-}=\frac{1+\mu_{V}}{2 f^{2}},
$$

where $f=94 \mathrm{MeV}$ is the pion decay constant and $\mu_{V} \simeq 3.7$.

From our previous analysis on meson scattering, we conjecture that the corrected amplitudes must satisfy

$$
A(s) \simeq A^{(0)}(s)+A^{(1)}(s)+O\left(\varepsilon^{2}\right) \text { for } s \simeq\left(m_{\pi}+m_{N}\right)^{2}
$$

where $\varepsilon$ is a small parameter characterizing the corrections and is typically of order $m_{\pi}^{2} / m_{\rho}^{2}$ and similarly for the $B$ amplitude.

By the optical theorem, one must have the first order corrected partial-wave satisfying

$$
\operatorname{Im} f_{I \ell}^{(1) \pm}(s)=|\vec{k}|\left|f_{I \ell}^{(0) \pm}(s)\right|^{2}
$$

The first order amplitude have the following imaginary parts:

$$
\begin{aligned}
& \operatorname{Im} F_{10}^{(1)}(s)=|\vec{k}| F_{10}^{(0)}(s)\left(F_{10}^{(0)}(s)+2 F_{21}^{(0)}(s)\right) \\
& \operatorname{Im} F_{20}^{(1)}(s)=|\vec{k}| F_{20}^{(0)}(s)\left(F_{20}^{(0)}(s)+2 F_{11}^{(0)}(s)\right) \\
& \operatorname{Im} F_{11}^{(1)}(s)=|\vec{k}| F_{11}^{(0) 2}(s) \\
& \operatorname{Im} F_{21}^{(1)}(s)=|\vec{k}| F_{21}^{(0) 2}(s)
\end{aligned}
$$

the amplitudes $f_{\ell I}^{ \pm(0)}$ follow from the projection of $A^{(0)}$ and $B^{(0)}$.

In order to compute the first order corrected Dirac amplitudes we construct the auxiliary functions $S, \mathcal{P}$ and $\mathcal{D}$, that are obtained from

$$
\begin{aligned}
& \operatorname{Im} \mathcal{S}(s)=\frac{2|\vec{k}|}{W} A_{0}^{(0)}(s), \quad \operatorname{Im} \mathcal{D}(s)=\frac{2|\vec{k}|}{W} B_{0}^{(0)}, \\
& \operatorname{Im} \mathcal{P}(s)=\frac{2|\vec{k}|}{W} A_{1}^{(0)}(s),
\end{aligned}
$$

by solving subtracted dispersion relation. This method introduces free parameters (called $\lambda$ ) and the final expression becomes:

$$
\begin{aligned}
& \mathcal{S}(s)=A_{0}^{(0)}(s) G(s), \\
& \mathcal{D}(s)=B_{0}^{(0)} G(s), \\
& \mathcal{P}(s)=\lambda_{1}+\lambda_{2} s+\lambda_{3} s^{2}+A_{1}^{(0)}(s) G(s),
\end{aligned}
$$

where

$$
\begin{array}{r}
G(s)=\frac{s^{3}}{\pi} \int_{(M+m)^{2}}^{\infty} \frac{\sqrt{R(x)}}{x^{4}(x-s)} d x, \\
R(s)=\left[s-(M+m)^{2}\right]\left[s-(M-m)^{2}\right] \\
X^{2}=M^{2}+m^{2}, \quad \text { and } x^{2}=M^{2}-m^{2},
\end{array}
$$


we obtain

$$
\begin{aligned}
& G(s)=1-\frac{X^{2}}{2 x^{4}} s+\frac{\sqrt{R(s)}}{s} \ln \frac{-s-X^{2}+\sqrt{R(s)}}{2 M m} \\
& +\left(\frac{1}{3}-\frac{X^{4}}{2 x^{4}}\right) \frac{s^{2}}{x^{4}}+\frac{1}{2 x^{10}} \ln \frac{M}{m}\left[X^{2}\left(X^{4}-x^{4}\right) s^{2}+\right. \\
& \left.+x^{4}\left(X^{4}-x^{4}\right) s+2 x^{8} X^{2}-\frac{2 x^{12}}{s}\right] .
\end{aligned}
$$

Expressing functions $A$ and $B$ in terms of Pauli amplitudes we construct auxiliary functions:

$$
\begin{aligned}
\mathcal{A}(s, t) & =\frac{1}{4}\left[a_{1}(s) \mathcal{S}+a_{2}(s) \mathcal{D}+3 \cos \theta a_{3}(s) \mathcal{P}\right] \\
\mathcal{B}(s, t) & =\frac{1}{4}\left[b_{1}(s) \mathcal{S}+b_{2}(s) \mathcal{D}+3 \cos \theta b_{3}(s) \mathcal{P}\right]
\end{aligned}
$$

where:

$$
\begin{aligned}
a_{1}(s) & =(W+M)\left(F_{10}^{(0)}+2 F_{21}^{(0)}\right) \\
& +(W-M)\left(F_{20}^{(0)}+2 F_{11}^{(0)}\right) \\
a_{2}(s) & =\left(W^{2}-M^{2}\right)\left(F_{10}^{(0)}+2 F_{21}^{(0)}-F_{20}^{(0)}-2 F_{11}^{(0)}\right), \\
a_{3}(s) & =(W+M) F_{11}^{(0)}+(W-M) F_{21}^{(0)}, \\
b_{1}(s) & =F_{10}^{(0)}+2 F_{21}^{(0)}-F_{20}^{(0)}-2 F_{11}^{(0)}, \\
b_{2}(s) & =(W-M)\left(F_{10}^{(0)}+2 F_{21}^{(0)}\right) \\
& +(W+M)\left(F_{20}^{(0)}+2 F_{11}^{(0)}\right) \\
b_{3}(s) & =F_{11}^{(0)}-F_{21}^{(0)} .
\end{aligned}
$$

At this point it starts the difference between the previous

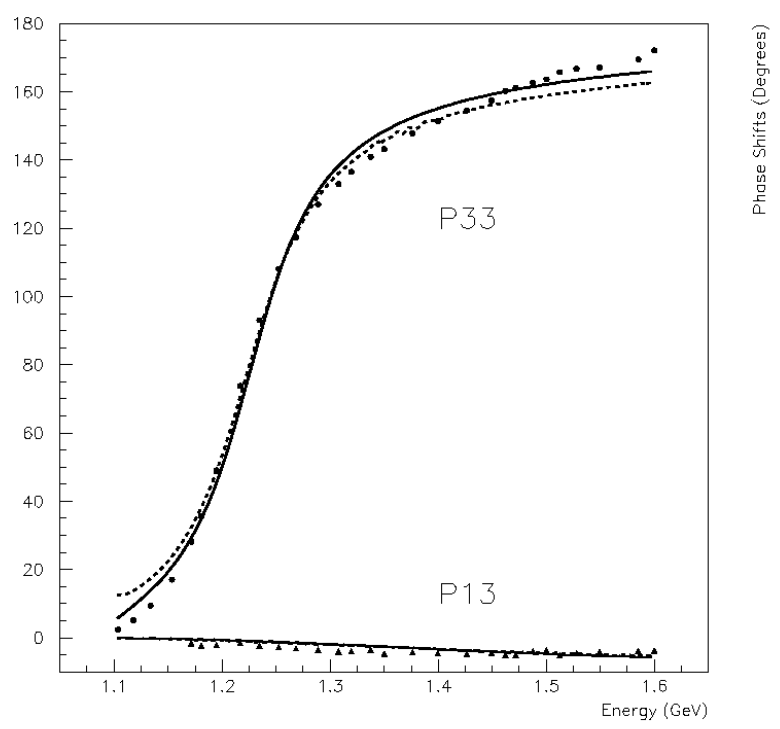

FIG. 1: $P_{33}$ phase-shifts as a function of center of mass energy. Dashed line corresponds to the fit performed in the previous analysis and the solid line corresponds to the present analysis (without crossing). The result for $P_{31}$ phase-shifts is also presented, with the same convention. Experimental data are from[5]. and the present work. In the previous work we have imposed crossing symmetry by constructing

$$
\begin{aligned}
& A^{ \pm}(s, t)=\mathcal{A}(s, t) \pm \mathcal{A}(u, t), \\
& B^{ \pm}(s, t)=\mathcal{B}(s, t) \mp \mathcal{B}(u, t),
\end{aligned}
$$

in the present exercise we do not add the second terms in the above expressions, in order to evaluate the importance of crossing symmetry constraint. Another difference is that in the previous work the free parameters were introduced also in the expressions of $\mathcal{S}$ and $\mathcal{D}$.

\section{RESULTS AND CONCLUSION}

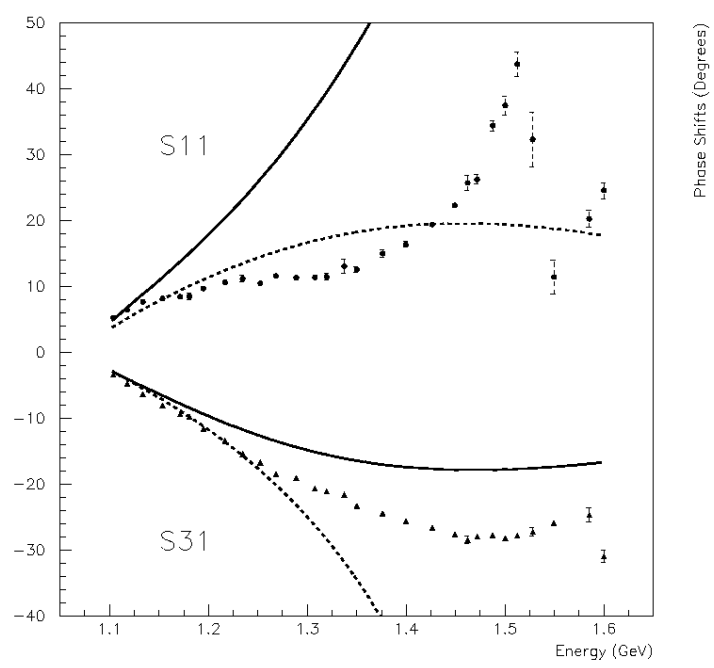

FIG. 2: The results for $S_{11}$ and $S_{31}$ phase-shifts as a function of center of mass energy. Dashed line corresponds to the previous result solid line corresponds to the present analysis (without crossing). Experimental data are from[5].

In the Fig. 1 we have plotted the fits of the amplitudes obtained by the two procedures to the experimental $P_{33}$ phaseshifts. Our first conclusion is that we need to introduce three free parameters $\left(\lambda_{1}=10,86, \lambda_{2}=-31,16\right.$ and $\left.\lambda_{3}=47,04\right)$ for the $P_{33}$ amplitude. Note that, in the previous analysis, by crossing, $P_{33}$ depends also on $\mathcal{S}$ and $\mathcal{D}$. In this figure we also show the resulting previous and present $P_{13}$ phase shifts.

In the Fig. 2 we show the resulting phase shifts for $S_{11}$ and $S_{31}$ and in the Fig. 3 those for $P_{11}$ and $P_{31}$ phase-shifts. It is clear from the plots that the quality of the adjust to the experimental phase shifts get worse when crossing symmetry is not imposed in the model amplitudes. We would like to mention that by the Inverse Amplitude Method [4], that respect exact unitarity while violates crossing symmetry, the adjust to the experimental phase shifts requires ten parameters.

In conclusion, in this work we have described a method to construct $\pi-N$ amplitudes which satisfy approximate unitarity, and we do not impose crossing symmetry on the total 


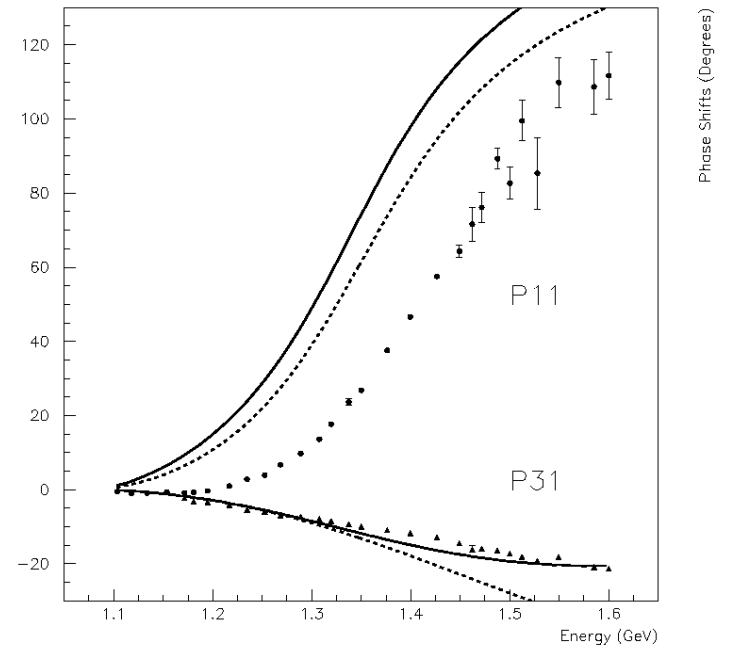

FIG. 3: The results for $P_{11}$ and $P_{13}$ phase-shifts as a function of center of mass energy. Dashed line corresponds to the previous result and the solid line corresponds to the present analysis (without crossing). Experimental data are from[5].

amplitudes. We follow the same procedure as in a previous work [2] by starting from a soft-pion representation, that re- produces pion-nucleon Weinberg $S$-wave scattering length, and we do not include single-particle pole contribution. As specific results, we have found that the fit of $P_{33}$ to the experimental phase shifts requires three parameters while only two parameters were used when working with a crossing symmetric amplitude. On the other hand the five resulting low energy phase shifts are quite far from the experimental points. We also observe that when $S_{31}$ gets closer to the the experimental phase-shifts, the $S_{11}$ becomes wrong and vice-versa. It is clear that this framework does not allow one to access the center of mass energies corresponding to the Figs. 1, 2 and 3. However, our aim is to compare our results with those published in the previous work [2], where we adopted this wide range for the center of mass energy.

To take a broader view, the power of the method can be traced directly to the expression of corrected amplitudes that allow one to reproduce the Delta resonance while leading to $S$ - and $P$-partial waves in qualitative agreement with data for very low energies. The model fails in the description of other resonant states as $\mathrm{N}(1440)$ and $\mathrm{N}(1535)$ and do not include addicional pion production that could be important for the description of some of the partial waves.

However, going to higher-order corrections would probably improve amplitude unitarity property. We believe that model second-order approximation permits $\ell=2$ resonances. Therefore only third-order will bring important $F$-wave and so on.
[1] J. R. Peláez, Phys. Rev. D 62, 017502 (2000).

[2] J. Sá Borges, Nucl. Phys. A 662, 362 (2000).

[3] J. Gasser and H. Leutwyler, Nucl. Phys. B 250, 465 (1985).

[4] N. Fettes, U. Meissner, M. Mojzis, and S. Steininger, Annals Phys. 283, 273 (2000).
[5] E. T. Osypowski, Nucl. Phys. B 21, 615 (1970).

[6] SAID on-line program, R. A. Arndt, R. L. Workmann et al., http://said.phys.vt.edu 